\title{
Comunicación
}

\section{Análisis retrospectivo de agentes bacterianos y patrones de susceptibilidad antibiótica en casos de infecciones del tracto urinario en caninos domésticos (2012-2017)}

\section{Retrospective analysis of microbial agents and antibiotic susceptibility patterns in cases of urinary tract infections in domestic canines (2012-2017)}

\author{
Milagros García M. ${ }^{1}$, Diego Díaz C. ${ }^{1}$, Carlos Huerta M. ${ }^{1}$, Juan Olazábal L. ${ }^{2}$, \\ Manuel Barrios-Arpi ${ }^{3}$, Ysaac Chipayo G. ${ }^{1,4}$
}

\section{Resumen}

\begin{abstract}
El presente estudio tuvo como objetivo describir y analizar los resultados de los aislados bacterianos y sus antibiogramas en pacientes caninos con diagnóstico de infección del tracto urinario, que fueron atendidos en la Clínica de Animales Menores de la Facultad de Medicina Veterinaria de la Universidad Nacional Mayor de San Marcos entre 2012 y 2017 . Se identificaron 97 historias clínicas de pacientes que contaban con exámenes de urocultivos y antibiogramas. Los agentes bacterianos aislados fueron Escherichia coli (36.9\%), Staphylococcus sp (28.7\%), Proteus sp (27.9\%), Pseudomonas $\mathrm{sp}(3.3 \%)$, Klebsiella $\mathrm{sp}(1.6 \%)$, Streptococcus $\mathrm{sp}(0.8 \%)$ y Citrobacter $\mathrm{sp}(0.8 \%)$. Estos evidenciaron una alta resistencia antibiótica debido posiblemente al uso inadecuado de los antibióticos en el medio, por lo que no permite recomendar un tratamiento inicial seguro en pacientes caninos con diagnóstico presuntivo de esta enfermedad.
\end{abstract}

Palabras clave: infección del tracto urinario; agente bacteriano; urocultivo; antibiograma; susceptibilidad antibiótica; resistencia antibiótica

\section{AbSTRaCT}

The present study aimed to describe and analyse the results of bacterial isolates and their antibiograms in canine patients with a diagnosis of urinary tract infection, which were treated at the Small Animal Clinic of the Faculty of Veterinary Medicine of

\footnotetext{
${ }^{1}$ Clínica de Animales Menores, Facultad de Medicina Veterinaria, Universidad Nacional Mayor de San Marcos, Lima, Perú

${ }^{2}$ Laboratorio de Bioquímica, Nutrición y Alimentación Animal, Facultad de Medicina Veterinaria, Universidad Nacional Mayor de San Marcos, Lima, Perú

${ }^{3}$ Laboratorio de Fisiología Animal, Facultad de Medicina Veterinaria, Universidad Nacional Mayor de San Marcos, Lima, Perú

${ }^{4}$ E-mail: ychipayog@unmsm.edu.pe
}

Recibido: 30 de enero de 2019

Aceptado para publicación: 20 de octubre de 2019 
Universidad de San Marcos between 2012 and 2017. In total, 97 medical records of patients who had urine cultures and antibiograms exams were identified. The bacterial agents isolated were Escherichia coli (36.9\%), Staphylococcus sp (28.7\%), Proteus sp (27.9\%), Pseudomonas sp (3.3\%), Klebsiella sp (1.6\%), Streptococcus sp (0.8\%) and Citrobacter $\mathrm{sp}(0.8 \%)$ These evidenced a high antibiotic resistance due possibly to the inappropriate use of antibiotics in the country, so it is not possible to recommend a safe initial treatment in canine patients with presumptive diagnosis of this disease.

Key words: urinary tract infection; bacterial agent; urine culture; antibiotic susceptibility; antibiotic resistance

\section{INTRODUCCIÓN}

La infección del tracto urinario (ITU) es el resultado de la adhesión, multiplicación y persistencia de un agente infeccioso (frecuentemente de origen bacteriano) en porciones del sistema urogenital, produciéndose como consecuencia de la migración ascendente de bacterias a través del tracto urogenital (Elliott y Grauer, 2007; Cortadellas, 2010; Chew et al., 2011; Gaymer, 2014).

Los agentes bacterianos mayormente reportados asociados a las infecciones del tracto urinario en el perro son Escherichia coli, Staphylococcus pseudintermedius, Staphylococcus aureus betahemolítico, Streptococcus sp, Proteus sp, Enterococcus $\mathrm{sp}$, Enterobacter sp, Klebsiella sp y Pseudomonas sp (Thompson et al., 2011; Greene, 2012; Nelson y Couto, 2014; Windahl, 2015). De estas, E. coli es la bacteria aislada con mayor frecuencia en la ITU canina, así como de la humana y felina (Greene, 2012; Nelson y Couto, 2014; Windahl, 2015), siendo alrededor de 33 a $55 \%$ de los casos, según Thompson et al. (2011).

La ITU es un cuadro clínico común en perros y poco frecuente en gatos (Chew et al., 2011; Ybarra et al., 2014; Windahl, 2015; Wong et al., 2015), siendo las hembras las más afectadas debido, probablemente, a su uretra más ancha y corta, favoreciendo la contaminación fecal e inoculación de microorganismos (Elliott y Grauer, 2007; Wong et al., 2015). En relación a la edad y la raza, se reporta la presentación de cuadros de infección urinaria entre los cuatro meses y los 17 años, siendo las razas mayormente afectadas el Pastor alemán, Caniche Miniatura y Toy, Labrador Retriever, Dachshund, Doberman Pinscher y Schnauzer Miniatura (Chew et al., 2011; Gaymer, 2014). La infección del tracto urinario se observa en pacientes con signos clínicos de distrés del tracto urinario inferior, tales como hematuria, polaquiuria, estranguria o disuria, micción en sitios inapropiados e incontinencia, pero también puede presentarse como una bacteriuria asintomática o subclínica (Chew et al., 2011; Weese et al., 2011; Windahl, 2015; Wong et al., 2015).

Chew et al. (2011) refiere que la ITU ocurre como episodio no recurrente en el $75 \%$ de los perros afectados; sin embargo, los episodios múltiples pueden ocurrir en algunos perros con factores predisponentes. De igual manera, Thompson et al. (2011) y Windahl (2015) describieron que el $4.5 \%$ de los perros presentaron ITU recurrente. Dentro de los factores predisponentes a ITU se incluyen: micción anormal, defectos anatómicos del tracto urinario, urotelio anormal, composición alterada de la orina o inmunidad alterada (Windahl, 2015). Las anomalías congénitas conllevan a una mayor susceptibilidad a infecciones (Chew et al., 2011). 


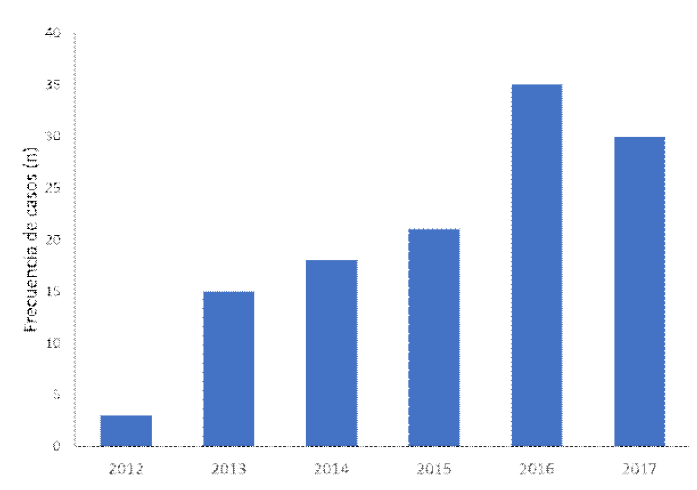

Figura 1. Frecuencia anual de casos de infecciones del tracto urinario en caninos atendidos en la Clínica de Animales Menores de la Universidad Nacional Mayor de San Mar$\cos (2012-2017)$

La terapia antimicrobiana empírica inicial está indicada en la mayoría de los casos mientras se espera los resultados de urocultivo y susceptibilidad antibiótica para aliviar la incomodidad del paciente, aunque el tratamiento antimicrobiano previo de la ITU podría tener un impacto en los patrones de resistencia de la microbiota residente del perro, facilitando el desarrollo de bacterias resistentes (Weese et al., 2011; Windahl, 2015). Uno de los factores a tener en cuenta al elegir el antimicrobiano debería ser la susceptibilidad reportada localmente de las presuntas bacterias involucradas (Weese et al., 2011).

El objetivo del presente estudio fue describir y analizar los resultados de urocultivos y los patrones de susceptibilidad antimicrobiana de pacientes caninos con diagnóstico de ITU en la Clínica de Animales Menores de la Facultad de Medicina Veterinaria de la Universidad Nacional de San Marcos durante el periodo 2012-2017.

\section{Materiales y Métodos}

Se evaluó la totalidad de historias clínicas de los pacientes caninos atendidos en la Clínica de Animales Menores (CAMe) de la
Facultad de Medicina Veterinaria (FMV) de la Universidad Nacional Mayor de San Marcos (UNMSM) durante los años 2012-2017. Se recopilaron las historias clínicas de los pacientes caninos con diagnóstico presuntivo de infección del tracto urinario y se utilizaron aquellas en las que se realizó urocultivo y antibiograma.

La información fue recopilada en una hoja de cálculo del programa Microsoft Office Excel $2016 \AA$, diseñándose un formato donde se ingresó la información del número de la historia clínica, nombre del paciente, procedencia, sexo, raza, edad, fecha de examen de urocultivo y resultado de urocultivo y antibiograma.

Se evaluaron las variables sexo, raza y edad. La edad se dividió en cinco categorías: $<1,1-4,5-8,9-12$ y $>12$ años (Solano, 2017). La frecuencia de los agentes bacterianos y los patrones de sensibilidad antibiótica fue establecida mediante la fórmula: $\mathrm{Ni}$ (total de valores $\left.\mathrm{d} \gg \mathrm{X}_{1}\right) / \mathrm{N}\left(\right.$ total de datos: $\left.\mathrm{X}_{1}, \mathrm{X}_{2}, \ldots \mathrm{X}_{\mathrm{n}}\right)$ $\mathrm{x} 100$.

\section{Resultados y Discusión}

Se recopilaron 29144 historias clínicas, de las cuales 314 correspondían a pacientes caninos con sospecha de ITU y 97 contaban con exámenes de urocultivo positivo y antibiograma. Esto representa el $0.33 \%$ del total y coincide con los resultados reportados por Gaymer (2014), quien encontró una frecuencia de ITU igual a $0.4 \%(n=48 / 12150)$ en el Hospital Veterinario de Alta Complejidad, sede Bilbao, de la Universidad de Chile. La frecuencia anual de infecciones del tracto urinario en caninos atendidos en la CAMe fue mayor durante los dos últimos años de evaluación (Figura 1) debido a la mayor afluencia de atención médica en CAMe, además de un mayor uso del urocultivo y del antibiograma como principales métodos para el diagnóstico y tratamiento de infecciones del tracto urinario. 
Cuadro 1. Distribución de la infección del tracto urinario de 97 casos según el rango de edad en caninos atendidos en la Clínica de Animales Menores de la Universidad Nacional Mayor de San Marcos (20122017)

\begin{tabular}{ccc}
\hline \multirow{2}{*}{ Edad (años) } & \multicolumn{2}{c}{ Frecuencia } \\
\cline { 2 - 3 } & $\mathrm{n}$ & $\%$ \\
\hline$<1$ & 1 & 1.0 \\
$1-4$ & 28 & 28.9 \\
$5-8$ & 25 & 25.8 \\
$9-12$ & 33 & 34.0 \\
$>12$ & 10 & 10.3 \\
\hline
\end{tabular}

Cuadro 2. Frecuencia de agentes bacterianos asociados a infecciones del tracto urinario en 122 cultivos en 97 caninos atendidos en la Clínica de Animales Menores de la Universidad Nacional Mayor de San Marcos (20122017)

\begin{tabular}{lcc}
\hline \multirow{2}{*}{ Bacteria } & \multicolumn{2}{c}{ Frecuencia } \\
\cline { 2 - 3 } & $\mathrm{n}$ & $\%$ \\
\hline Escherichia coli & 45 & 36.9 \\
Staphylococcus $\mathrm{sp}$ & 35 & 28.7 \\
Proteus $\mathrm{sp}$ & 34 & 27.8 \\
Pseudomonas $\mathrm{sp}$ & 4 & 3.3 \\
Klebsiella $\mathrm{sp}$ & 2 & 1.6 \\
Streptococcus $\mathrm{sp}$ & 1 & 0.8 \\
Citrobacter $\mathrm{sp}$ & 1 & 0.8 \\
\hline
\end{tabular}

La frecuencia de ITU fue mayor en hembras (53\%) que en machos (47\%), coincidiendo con otros reportes (Papini et al., 2006; Windahl, 2015; Wong et al., 2015), debido posiblemente a una uretra más gruesa y corta en hembras que facilita el ascenso de las bacterias hacia la vejiga; así mismo, los machos poseen un mecanismo protector adicional por las secreciones prostáticas que presentan propiedades antimicrobianas (Elliott y Grauer, 2007).

La mayor frecuencia de ITU se halló en caninos cruzados (38.1\%), seguido por las razas Schnauzer (17.5\%), Shih Tzu (7.2\%) y Cocker Spaniel $(6.2 \%)$. No existen datos comparativos para perros mestizos, toda vez que la mayoría de los estudios incluye solamente animales de raza; sin embargo, la mayoría de los pacientes en CAMe son mestizos. En cuanto animales de raza, la frecuencia encontrada para la raza Schnauzer coincide con lo descrito por Chew et al. (2011), quienes la reportan como una de las razas con mayor incidencia de esta enfermedad.

La edad no fue una variable de relevancia en la frecuencia de presentación de ITU, pues perros con rangos de edades 1-4, 5-8 y 9-12 tuvieron presentaron frecuencias similares (Cuadro 1). Chew et al. (2011) consideran una edad media de presentación de 7 años, y Thompson et al. (2011) sugieren una edad de presentación entre 7 y 8 años, mientras que Gaymer (2014) refiere un rango de edad entre 3 a 17 años, con una edad media de 8.5 años. Estos resultados confirman que la ITU se relaciona mayormente con la edad adulta, posiblemente debido a una disminución del sistema inmune, el cual altera los mecanismos de defensa del hospedero (Elliot y Grauer, 2007).

De las 97 historias clínicas se obtuvo un total de 122 urocultivos (esto debido a infecciones recurrentes), donde se evidenciaron siete agentes bacterianos asociados con mayor frecuencia a las infecciones del tracto urinario (Cuadro 2). E. coli fue la bacteria aislada con mayor frecuencia (36.9\%), coincidiendo con el estudio de Thompson et al. (2011), donde E. coli representó el 33\% de los casos, y con Marques et al. (2016), quienes reportan tasas mayores de $30 \%$ de los casos. Incluso, Rubin y Gaunt (2011) reportaron que el $51 \%$ de los casos fue debido a E. coli. Esta bacteria es considerada un componente normal de la flora urogenital distal y gastrointestinal, pudiendo ascender por la ure- 
Cuadro 3. Sensibilidad de E. coli frente a los antibióticos más frecuentemente utilizados en infecciones del tracto urinario en caninos atendidos en la Clínica de Animales Menores de la Universidad nacional Mayor de San Marcos, Lima (2012-2017)

\begin{tabular}{lccccccc}
\hline \multirow{2}{*}{ Antibiótico } & \multicolumn{9}{c}{ Condición } \\
\cline { 2 - 9 } & $\begin{array}{c}\mathrm{N} .^{\circ} \text { de } \\
\text { pruebas }\end{array}$ & $\mathrm{n}$ & $\%$ & $\mathrm{n}$ & $\%$ & $\mathrm{n}$ & $\%$ \\
\hline Amikacina & 34 & 27 & 79.4 & 2 & 5.9 & 5 & 14.7 \\
Amoxicilina + & & 18 & 47.4 & 5 & 13.2 & 15 & 39.5 \\
ácido clavulánico & 38 & & & & & \\
Ceftriaxona & 31 & 17 & 54.8 & 2 & 6.5 & 12 & 38.7 \\
Ciprofloxacina & 39 & 19 & 48.7 & 6 & 15.4 & 14 & 35.9 \\
Ácido nalidíxico & 12 & 1 & 8.3 & 2 & 16.7 & 9 & 75.0 \\
Cloranfenicol & 14 & 3 & 21.4 & - & - & 11 & 78.6 \\
Doxiciclina & 17 & 5 & 29.4 & - & - & 12 & 70.6 \\
Enrofloxacina & 40 & 9 & 22.5 & 1 & 2.5 & 30 & 75.0 \\
Gentamicina & 33 & 12 & 36.4 & 7 & 21.2 & 14 & 42.4 \\
Nitrofurantoina & 29 & 9 & 31.0 & 2 & 6.9 & 18 & 62.1 \\
Oxitetraciclina & 12 & 3 & 25.0 & 2 & 16.7 & 7 & 58.3 \\
Sulfatrimetroprim & 41 & 15 & 36.6 & 5 & 12.2 & 21 & 51.2 \\
\hline
\end{tabular}

tra e ingresar al tracto urinario (Cortadellas, 2010). Staphylococcus sp representó una frecuencia de $28.7 \%$, seguida de Proteus sp (27.9\%), lo cual coincide con otros autores (Greene, 2012; Nelson y Couto, 2014; Windahl, 2015).

Se determinaron patrones de susceptibilidad, especialmente para los tres uropatógenos más comunes. Los antibióticos de mayor susceptibilidad para E. coli fueron amikacina (79.4\%), ceftriaxona (54.8\%), ciprofloxacina $(48.7 \%)$ y amoxicilina más ácido clavulánico (47.4\%) (Cuadro 3), difiriendo con lo reportado por Gaymer (2014), quien describe mayor susceptibilidad a la amoxicilina más ácido clavulánico (85\%) en Chile, así como de reportes en otras localidades (Chew et al., 2011; Weese et al., 2011). Con respecto a Staphylococcus sp, la mayor sensibilidad fue observada con amoxicilina con ácido clavulánico (89.4\%), cefalexina (64.3\%) y ceftriaxona (63.6\%) (Cuadro 4), coincidiendo con lo reportado por Gaymer
(2014). Para el caso de Proteus sp, los antibióticos con mayor susceptibilidad fueron ceftriaxona $(72.7 \%)$, amikacina $(70.4 \%)$, ciprofloxacina $(62.1 \%)$ y sulfatrimetoprim (55.6\%) (Cuadro 5), coincidiendo con Féria et al. (2002) y Schaffer y Pearson (2015), en donde la terapia de primera línea frente a esta bacteria consistió en el uso de sulfatrimetoprim, fluoroquinolonas y nitrofurantoína.

Los antibióticos con mayor efecto antimicrobiano en las tres cepas bacterianas más comúnmente aisladas fueron la amikacina y ceftriaxona, lo cual es coincidente con otros reportes (Thompson et al., 2011; Greene, 2012; Nelson y Couto, 2014; Windahl, 2015). Sin embargo, los otros datos de sensibilidad antibiótica resultaron diferente al reportado por estos autores, posiblemente debido al uso inadecuado y hasta indiscriminado de los antibióticos para el tratamiento de otras patologías en el país, lo cual conlleva a la alteración de la microbiota normal del hospedero 
Cuadro 4. Sensibilidad de Staphylococcus sp frente a los antibióticos más frecuentemente utilizados en infecciones del tracto urinario en caninos atendidos en la Clínica de Animales Menores de la Universidad nacional Mayor de San Marcos, Lima (2012-2017)

\begin{tabular}{lccccccc}
\hline \multirow{2}{*}{ Antibiótico } & \multicolumn{8}{c}{ Condición } \\
\cline { 2 - 9 } & $\begin{array}{c}\mathrm{N} .^{\circ} \text { de } \\
\text { pruebas }\end{array}$ & \multicolumn{2}{c}{ Sensible } & \multicolumn{2}{c}{ Intermedio } & \multicolumn{2}{c}{ Resistente } \\
\hline Amoxicilina + & 19 & 17 & 89.5 & 1 & 5.3 & 1 & 5.3 \\
ácido clavulánico & 19 & 9 & 64.3 & 1 & 7.1 & 4 & 28.6 \\
Cefalexina & 14 & 9 & $\mathrm{n}$ & $\%$ & $\mathrm{n}$ & $\%$ \\
Ceftriaxona & 11 & 7 & 63.6 & 1 & 9.1 & 3 & 27.3 \\
Ciprofloxacina & 14 & 7 & 50.0 & 1 & 7.1 & 6 & 42.9 \\
Clindamicina & 11 & 4 & 36.4 & 1 & 9.1 & 6 & 54.6 \\
Enrofloxacina & 20 & 8 & 40.0 & 0 & - & 12 & 60.0 \\
Gentamicina & 13 & 5 & 38.5 & 1 & 7.7 & 7 & 53.9 \\
Sulfatrimetoprim & 21 & 4 & 19.0 & 5 & 23.8 & 12 & 57.1 \\
\hline
\end{tabular}

Cuadro 5. Sensibilidad de Proteus sp frente a los antibióticos más frecuentemente utilizados en infecciones del tracto urinario en caninos atendidos en la Clínica de Animales Menores de la Universidad nacional Mayor de San Marcos, Lima (2012-2017)

\begin{tabular}{lccccccc}
\hline \multirow{2}{*}{ Antibiótico } & \multicolumn{9}{c}{ Condición } \\
\cline { 2 - 8 } & $\begin{array}{c}\text { N. }{ }^{\circ} \text { de } \\
\text { pruebas }\end{array}$ & \multicolumn{2}{c}{ Sensible } & $\%$ & \multicolumn{2}{c}{ Intermedio } & \multicolumn{2}{c}{ Resistente } \\
\hline Amikacina & 27 & 19 & 70.4 & 1 & 3.7 & 7 & 25.9 \\
Amoxicilina + & 29 & 13 & 44.8 & 4 & 13.8 & 12 & 41.4 \\
ácido clavulánico & & & & & & \\
Ceftriaxona & 22 & 16 & 72.7 & - & - & 6 & 27.3 \\
Ciprofloxacina & 29 & 18 & 62.1 & 3 & 10.3 & 8 & 27.6 \\
Sulfatrimetroprim & 27 & 15 & 55.6 & 1 & 3.7 & 11 & 40.7 \\
Cefalotina & 10 & - & - & 1 & 10.0 & 9 & 90.0 \\
Enrofloxacina & 27 & 10 & 37.0 & 3 & 11.1 & 14 & 51.9 \\
Gentamicina & 20 & 7 & 35.0 & 3 & 15.0 & 10 & 50.0 \\
Nitrofurantoína & 23 & 5 & 21.7 & 5 & 21.7 & 13 & 56.5 \\
\hline
\end{tabular}


y aparición de cepas resistentes. Esto demuestra la necesidad de instaurar protocolos de diagnóstico, evaluación y tratamiento de los pacientes con sospecha de infección urinaria en base a los resultados del urocultivo y antibiograma, a fin de lograr un tratamiento específico y exitoso.

\section{Conclusiones}

- Escherichia coli, Staphylococcus sp y Proteus sp fueron los agentes bacterianos de mayor presentación en los casos de infección del tracto urinario (ITU) en caninos atendidos en la Clínica de Animales Menores de la Universidad Nacional Mayor de San Marcos durante los años 2012-2017.

- Los principales agentes bacterianos asociados a esta enfermedad mostraron mayor sensibilidad frente a amikacina y ceftriaxona.

\section{Literatura Citada}

1. Chew D, Dibartola S, Schenck P. 2011. Canine and feline nephrology and urology. $2^{\mathrm{a}}$ ed. Missouri: Elsevier. $526 \mathrm{p}$.

2. Cortadellas O. 2010. Manual de nefrología y urología clínica canina y felina. Zaragoza: Servet. 246 p.

3. Elliott J, Grauer G. 2007. BSAVA Manual of canine and feline nephrology and urology. $2^{\mathrm{a}}$ ed. Dorset: British Small Animal Veterinary Association. 298 p.

4. Féria C, Correia J, Machado J, Vidal $\boldsymbol{R}$, Gonçalves J. 2002. Urinary tract infection in dogs. Boston: Springer. $867 \mathrm{p}$.

5. Gaymer E. 2014. Descripción de registros clínicos de perros y gatos con infecciones del tracto urinario (ITU). Tesis de Médico Veterinario. Santiago: Univ. de Chile. 39 p.

6. Greene C. 2012. Infectious diseases of the dog and cat. $4^{\mathrm{a}}$ ed. Missouri: Elsevier. $1354 \mathrm{p}$.
7. Marques C, Telo L, Belas A, Bergström K, Beurlet S, Briend-Marchal A, Broens $E$, et al. 2016. European multicenter study on antimicrobial resistance in bacteria isolated from companion animal urinary tract infections. BMC Vet Res 12(213). doi: 10.1186/s12917-016-0840-3

8. Nelson R, Couto C. 2014. Small animal internal medicine. $5^{\mathrm{a}}$ ed. Missouri: Elsevier. $1473 \mathrm{p}$.

9. Papini R. Ebani V, Cerri D, Guidi G 2006. Survey on bacterial isolates from dogs with urinary tract infections and their in vitro sensitivity. Revue Méd Vét 157:35-41.

10. Rubin J, Gaunt M. 2011. Urinary tract infection caused by methicillin-resistant Staphylococcus pseudintermedius in a dog. Can Vet J 52: 162-164.

11. Schaffer J, Pearson M. 2015. Proteus mirabilis and urinary tract infections. Microbiol Spectr 3: 1-39. Doi: 10.1128/ microbiolspec.UTI-0017-2013

12. Solano N. 2017. Frecuencia de casos de piometría atendida en la Clínica de Animales Menores de la Facultad de Medicina Veterinaria de la UNMSM durante el período 2009-2013. Tesis de Médico Veterinario. Lima: Univ. Nacional Mayor de San Marcos. 40 p.

13. Thompson M, Litster A, Platell J, Trott D. 2011. Canine bacterial urinary tract infections: New developments in old pathogens. Vet J 190: 22-27. doi: 10.1016/ j.tvj1.2010.11.013

14. Weese J, Blondeau J, Boothe D, Breitschwerdt E, Guardabassi L, Hillier A, Lloyd D, et al. 2011. Antimicrobial use guidelines for treatment of urinary tract disease in dogs and cats: antimicrobial guidelines working group of the International Society for Companion Animal Infectious Diseases. Vet Med Int 2011:263768. doi: 10.4061/ 2011/263768

15. Windahl $U$. 2015. Bacterial infections in dogs with special reference to urinary- 
tract infections, surgical site infections and Methicillin-resistant Staphylococcus pseudintermedius. Acta Universitatis Agriculturae Sueciae. Uppsala, Sweden: SLU. 90 p.

16. Wong C, Epstein S, Westropp J. 2015. Antimicrobial susceptibility patterns inurinary tract infection in dogs (2010-
2013). J Vet Intern Med 29: 1045-1052. doi: 10.1111 jvim. 13571

17. Ybarra W, Skyes J, Wang Y, Byrne B, Westropp J. 2014. Performance of a veterinary urine dipstick paddle system for diagnosis and identification of urinary tract infections in dogs and cats. JAVMA 244: 814-819. doi: 10.2460/javma.244.7.814. 